Supporting information for

\title{
First-principles Study of the Ligand Substituent Effect on ORR Catalysis by Metallocorroles
}

\author{
Samala Nagaprasad Reddy, Chethana Bhadravathi Krishnamurthy and Ilya Grinberg
}

Department of Chemistry, Bar-Ilan University, Ramat Gan, Israel 52900

\section{Contents:}

\begin{tabular}{lll} 
Item & Description & Pages \\
\hline Table S1, S2 \& S3 & $\begin{array}{l}\text { Minimized energies of Mn- and Fe-corroles and energy differences of } \\
\text { singlet and triplet electronic states with respect to the minimized spin state }\end{array}$ & S2 \\
\hline Table S4, S5 \& S6 & $\begin{array}{l}\text { Minimized energies of Fe- and Co-corroles and energy differences of } \\
\text { singlet and triplet electronic states with respect to the minimized spin state }\end{array}$ & S3 \\
\hline Table S7 & E $_{\text {ads }}$ energies of Mn corroles in various spin states & S4 \\
\hline
\end{tabular}


Table S1: Converged energies of Mn-corroles, in various multiplicities using various ligands, data obtained from QE and in Ry units.

\begin{tabular}{|l|r|r|r|r|r|}
\hline system Mn & \multicolumn{1}{|l|}{ Corrole } & \multicolumn{1}{l|}{ Imidazole } & \multicolumn{1}{l}{ CH3 } & \multicolumn{1}{l|}{ CF3 } \\
\hline Singlet & & & & \\
\hline Corrole & -522.6914363893 & -523.888792005 & -600.985853584 & -537.7699972072 & -680.0849961292 \\
\hline${ }^{*} \mathrm{OOH}$ & -588.3917531818 & -589.46903091 & -666.5153261181 & -603.3149803247 & -745.6331645018 \\
\hline${ }^{*} \mathrm{O}$ & -555.1121898608 & -556.137058537 & -633.2024898174 & -569.9925844845 & -712.317234316 \\
\hline${ }^{*} \mathrm{OH}$ & -556.262611031 & -557.3649094722 & -634.4010270386 & -571.2076679091 & -713.5224680334 \\
\hline Triplet & & & & \\
\hline Corrole & -522.7522365944 & -523.949584944 & -601.032711019 & -537.8291996828 & -680.1456043187 \\
\hline${ }^{*} \mathrm{OOH}$ & -588.3947252545 & -589.5010602042 & -666.5758366173 & -603.3639153663 & -745.6860070767 \\
\hline${ }^{*} \mathrm{O}$ & -555.059401761 & -556.11430488 & -633.1889229778 & -569.985395131 & -712.2987959373 \\
\hline${ }^{*} \mathrm{OH}$ & -556.2959140058 & -557.3974813861 & -634.4755639927 & -571.2604366451 & -713.5837766364 \\
\hline $\mathrm{Optimized} \mathrm{spin}$ & & & & & \\
\hline $\mathrm{Corrole}$ & -522.8453777513 & -523.9841871696 & -601.059193853 & -537.8638070142 & -680.1743751606 \\
\hline${ }^{*} \mathrm{OOH}$ & -588.4177729644 & -589.5016407631 & -666.575309975 & -603.3640689211 & -745.6861955141 \\
\hline${ }^{*} \mathrm{O}$ & -555.1125672329 & -556.1389168367 & -633.2028490902 & -570.0154594436 & -712.3293772699 \\
\hline${ }^{*} \mathrm{OH}$ & -556.3195554117 & -557.3976037059 & -634.4747633281 & -571.2606658798 & -713.5839617218 \\
\hline
\end{tabular}

Table S2: Energy difference of singlet and triplet electronic states of Mn-corroles with respect to the optimized spin state, data obtained from QE and is in $\mathrm{eV}$.

\begin{tabular}{|l|l|l|r|r|r|}
\hline system Mn & Corrole & H & Imidazole & CH3 & CF3 \\
\hline Singlet & & & & & \\
\hline Corrole & 2.094479691226 & 1.297917806493 & 0.997845556086 & 1.276347909661 & 1.216064114650 \\
\hline${ }^{*} \mathrm{OOH}$ & 0.354017305795 & 0.443679815251 & 0.816122245809 & 0.667884621098 & 0.721523941481 \\
\hline${ }^{*} \mathrm{O}$ & 0.005134410851 & 0.025283464633 & 0.004888157239 & 0.311229786784 & 0.165213364390 \\
\hline${ }^{*} \mathrm{OH}$ & 0.774768050353 & 0.444827872218 & 1.003233691437 & 0.721074387448 & 0.836664557330 \\
\hline Triplet & & & & \\
\hline Corrole & 1.26725045829 & 0.470787433921 & 0.360317443333 & 0.470856901895 & 0.391447387993 \\
\hline${ }^{*} \mathrm{OOH}$ & 0.313580182009 & 0.007898909102 & -0.00716533612 & 0.002089220245 & 0.002563822368 \\
\hline${ }^{*} \mathrm{O}$ & 0.723353358202 & 0.334862851671 & 0.189474480545 & 0.409045959793 & 0.416080377807 \\
\hline${ }^{*} \mathrm{OH}$ & 0.321657830528 & 0.001664246265 & -0.01089360079 & 0.003118898114 & 0.002518216069 \\
\hline
\end{tabular}

Table S3: Converged energies of Fe-corroles, in various multiplicities using various ligands, data obtained from QE and in Ry units.

\begin{tabular}{|l|l|l|l|r|r|}
\hline system Fe & Corrole & H & Imidazole & \multicolumn{1}{l}{ CH3 } & \multicolumn{1}{l}{ CF3 } \\
\hline Singlet & & & & \\
\hline Corrole & -561.8478087245 & -563.0367302403 & -640.1017744299 & -576.9051903272 & -719.2289237348 \\
\hline${ }^{*} \mathrm{OOH}$ & -627.4780652507 & -628.5810792893 & -705.6337949212 & -642.433185969 & -784.7573830952 \\
\hline${ }^{*} \mathrm{O}$ & -594.1256651716 & -595.1945868107 & -672.2410610005 & -609.0452516102 & -751.3643938315 \\
\hline${ }^{*} \mathrm{OH}$ & -595.3565686366 & -596.4646129483 & -673.5137404951 & -610.3139936616 & -752.6333518196 \\
\hline Doublet & & & & \\
\hline Corrole & -561.8771939757 & -563.0552910739 & -640.119976096 & -576.9268602685 & -719.2463484088 \\
\hline${ }^{*} \mathrm{OOH}$ & -627.4836628114 & -628.5957148308 & -705.6549578414 & -642.45112884 & -784.7722113794 \\
\hline${ }^{*} \mathrm{O}$ & -594.1403732072 & -595.2000968042 & -672.2486065856 & -609.0556242608 & -751.3767547273 \\
\hline${ }^{*} \mathrm{OH}$ & -595.3739129615 & -596.4899962675 & -673.549295411 & -610.3451879105 & -752.6694154697 \\
\hline Quartet & & & & \\
\hline Corrole & -561.9195655818 & -563.0217770515 & -640.1188424919 & -576.9015341927 & -719.2233948378 \\
\hline${ }^{*} \mathrm{OOH}$ & -627.4743145488 & -628.5889006818 & -705.6573941349 & -642.4480643902 & -784.7792712277 \\
\hline${ }^{*} \mathrm{O}$ & -594.1196526192 & -595.1800748759 & -672.2624938178 & -609.0443039523 & -751.3669648139 \\
\hline${ }^{*} \mathrm{OH}$ & -595.3759540454 & -596.4886058172 & -673.5511534872 & -610.3465896976 & -752.6704944363 \\
\hline Optimized spin & & & & & \\
\hline Corrole & -561.9195710577 & -563.0728666557 & -640.1407552085 & -576.9504533958 & -719.2600178476 \\
\hline${ }^{*} \mathrm{OOH}$ & -627.497116668 & -628.5890696442 & -705.6572939382 & -642.451265245 & -784.7723736913 \\
\hline${ }^{*} \mathrm{O}$ & -594.1404261521 & -595.2022340314 & -672.2624026777 & -609.0585242902 & -751.3782626964 \\
\hline${ }^{*} \mathrm{OH}$ & -595.3977992645 & -596.4900859608 & -673.5511676775 & -610.3453396063 & -752.668657695 \\
\hline
\end{tabular}


Table S4: Energy difference of singlet, doublet and quartet electronic states of Fe-corroles with respect to the optimized spin state, data obtained from $\mathrm{QE}$ and is in $\mathrm{eV}$.

\begin{tabular}{|l|l|l|l|r|r|}
\hline system Mn & Corrole & H & Imidazole & CH3 & CF3 \\
\hline Singlet & & & & & \\
\hline Corrole & 0.976376638022 & 0.491661157115 & 0.530360704004 & 0.615835644907 & 0.423057110384 \\
\hline${ }^{*} \mathrm{OOH}$ & 0.259207831511 & 0.108714356209 & 0.319720530148 & 0.245981170505 & 0.203957524364 \\
\hline${ }^{*} \mathrm{O}$ & 0.200833443839 & 0.104045775888 & 0.290368416202 & 0.180584076605 & 0.188695588346 \\
\hline${ }^{*} \mathrm{OH}$ & 0.560971474275 & 0.346578116904 & 0.509222943190 & 0.426483459178 & 0.480361080644 \\
\hline Doublet & & & & \\
\hline Corrole & 0.576569782604 & 0.239128059302 & 0.282714330752 & 0.321000966473 & 0.185982257042 \\
\hline${ }^{*} \mathrm{OOH}$ & 0.183049110721 & -0.09041240247 & 0.031784227714 & 0.002072977761 & 0.002208366703 \\
\hline${ }^{*} \mathrm{O}$ & 0.000720352322 & 0.029078467981 & 0.187705463602 & 0.039456924399 & 0.020516972268 \\
\hline${ }^{*} \mathrm{OH}$ & 0.324989826529 & 0.001220339957 & 0.025473492697 & 0.002063927251 & -0.01031005377 \\
\hline Quartet & & & & \\
\hline Corrole & $7.45034408 \mathrm{E}-05$ & 0.695109729051 & 0.298137805863 & 0.665579907003 & 0.498281613603 \\
\hline${ }^{*} \mathrm{OOH}$ & 0.310238749098 & 0.002298851397 & -0.00136324604 & 0.043549863960 & -0.09384579765 \\
\hline${ }^{*} \mathrm{O}$ & 0.282638416398 & 0.301490779128 & -0.00124002468 & 0.193477623863 & 0.153715578079 \\
\hline${ }^{*} \mathrm{OH}$ & 0.297219455257 & 0.020138386916 & 0.000193068936 & -0.01700836478 & -0.02499014755 \\
\hline
\end{tabular}

Table S5: Converged energies of Co-corroles, in various multiplicities using various ligands, data obtained from QE and in Ry units.

\begin{tabular}{|c|c|c|c|c|c|}
\hline system Co & Corrole & $\mathrm{H}$ & Imidazole & CH3 & CF3 \\
\hline \multicolumn{6}{|l|}{ Singlet } \\
\hline Corrole & -610.0279750915 & -611.2113424016 & -688.2483564031 & -625.0728611593 & -767.3991424763 \\
\hline$* \mathrm{OOH}$ & -675.6024183892 & -676.7265309434 & -753.769355048 & -690.5805466942 & -832.9046983881 \\
\hline$* \mathrm{O}$ & -642.1758932928 & -643.264857421 & -720.3154408314 & -657.1211216921 & -799.4395459276 \\
\hline$* \mathrm{OH}$ & -643.4842413861 & -644.6024392582 & -721.6537383242 & -658.4573028897 & -800.7822207495 \\
\hline \multicolumn{6}{|l|}{ Triplet } \\
\hline Corrole & -610.0558402249 & -611.1517577468 & -688.2272432147 & -625.0201468518 & -767.3489555632 \\
\hline$* \mathrm{OOH}$ & -675.5872452777 & -676.7288082621 & -753.7750257424 & -690.5836415892 & -832.9097458539 \\
\hline$* \mathrm{O}$ & -642.2238164863 & -643.2983083406 & -720.3520624821 & -657.1558743756 & -799.4763959374 \\
\hline$* \mathrm{OH}$ & -643.4771417267 & -644.6016401471 & -721.6568090134 & -658.4572040586 & -800.7830467111 \\
\hline \multicolumn{6}{|c|}{ Optimized spin } \\
\hline Corrole & -610.0558540086 & -611.2130037243 & -688.2494115149 & -625.0757963424 & -767.3996396808 \\
\hline$* \mathrm{OOH}$ & -675.6090536793 & -676.728922319 & -753.7752456037 & -690.5827538406 & -832.9084551646 \\
\hline$* \mathrm{O}$ & -642.2238164973 & -643.2984255045 & -720.3527878623 & -657.1563179436 & -799.4777676284 \\
\hline$* \mathrm{OH}$ & -643.4932670734 & -644.6036481609 & -721.6576301645 & -658.4594121297 & -800.7844224483 \\
\hline
\end{tabular}

Table S6: Energy difference of singlet and Triplet electronic states of Co-corroles with respect to the optimized spin state, data obtained from QE and is in $\mathrm{eV}$.

\begin{tabular}{|l|r|r|r|r|r|}
\hline system Co & \multicolumn{1}{|l|}{ Corrole } & H & \multicolumn{1}{l|}{ Imidazole } & \multicolumn{1}{l}{ CH3 } & \multicolumn{1}{l|}{ CF3 } \\
\hline Singlet & & & & & \\
\hline Corrole & 0.379312128466 & 0.022603455047 & 0.014355532575 & 0.039935215027 & 0.006764814303 \\
\hline${ }^{*} \mathrm{OOH}$ & 0.090277753680 & 0.032536334376 & 0.080145122295 & 0.030029767505 & 0.051113566760 \\
\hline${ }^{*} \mathrm{O}$ & 0.652028650778 & 0.456717208751 & 0.508132426083 & 0.478869570960 & 0.520032920650 \\
\hline${ }^{*} \mathrm{OH}$ & 0.122800776240 & 0.016447965127 & 0.052951204041 & 0.028697682588 & 0.029955649105 \\
\hline Triplet & & & & \\
\hline Corrole & 0.000187536861 & 0.833294277617 & 0.301615199154 & 0.757150166626 & 0.689592800801 \\
\hline${ }^{*} \mathrm{OOH}$ & 0.296718527469 & 0.00155182374 & 0.00299136646 & -0.01207843941 & -0.01756072891 \\
\hline${ }^{*} \mathrm{O}$ & $1.49662642 \mathrm{E}-07$ & 0.001594096647 & 0.009869303984 & 0.006035052280 & 0.018662813586 \\
\hline${ }^{*} \mathrm{OH}$ & 0.219396598408 & 0.027320429473 & 0.011172333933 & 0.030042348694 & 0.018717864960 \\
\hline
\end{tabular}


Table S7: Adsorption energies $\left(\mathrm{E}_{\mathrm{ad}}\right)$ of ORR intermediates in various spin states of Mn-corroles in the gas phase obtained with QE using PBE functional, with and with out excess ligand L. All data is in $\mathrm{eV}$.

\begin{tabular}{|c|c|c|c|c|c|}
\hline$E_{a d}$ energies & Corrole & $\mathrm{H}$ & Imidazole & CH3 & CF3 \\
\hline \multicolumn{6}{|l|}{ Singlet } \\
\hline$* \mathrm{OOH}$ & 1.8843 & 3.5181 & 4.2088 & 3.9978 & 3.9544 \\
\hline$* \mathrm{O}$ & -1.1416 & 1.2052 & 1.6355 & 1.5546 & 1.4233 \\
\hline$* \mathrm{OH}$ & -0.9416 & 0.3517 & 1.1809 & 0.8748 & 0.8775 \\
\hline \multicolumn{6}{|l|}{ Triplet } \\
\hline$* \mathrm{OOH}$ & 2.6711 & 3.9094 & 4.0230 & 4.1374 & 4.0601 \\
\hline$* \mathrm{O}$ & 0.4038 & 2.3419 & 2.4576 & 2.4579 & 2.4988 \\
\hline$* \mathrm{OH}$ & -0.5675 & 0.7356 & 0.8043 & 0.9623 & 0.8680 \\
\hline \multicolumn{6}{|c|}{ Optimized spin } \\
\hline$* \mathrm{OOH}$ & 3.6248 & 4.3723 & 4.3905 & 4.6062 & 4.4490 \\
\hline$* \mathrm{O}$ & 0.9478 & 2.4778 & 2.6285 & 2.5197 & 2.4741 \\
\hline$* \mathrm{OH}$ & 0.3781 & 1.2048 & 1.1755 & 1.4301 & 1.2569 \\
\hline
\end{tabular}

